A N N A L E S Annales de Bretagne et des Pays de l'Ouest

Anjou. Maine. Poitou-Charente. Touraine

$108-2$ | 2001

Varia

\title{
Genre et pouvoir en cinéma
}

Notorious (1946), Eyes Wide Shut (1999) ou de l'enchaînement des

sentiments au déchaînement du désir

\section{François Rouquet}

\section{(2) OpenEdition}

\section{Journals}

Édition électronique

URL : http://journals.openedition.org/abpo/1742

DOI : $10.4000 / a b p o .1742$

ISBN : 978-2-7535-1482-9

ISSN : 2108-6443

Éditeur

Presses universitaires de Rennes

Édition imprimée

Date de publication : 20 juin 2001

Pagination : 129-155

ISBN : 978-2-86847-635-7

ISSN : 0399-0826

\section{Référence électronique}

François Rouquet, « Genre et pouvoir en cinéma », Annales de Bretagne et des Pays de l'Ouest [En ligne], 108-2 | 2001, mis en ligne le 20 juin 2003, consulté le 21 avril 2019. URL : http:// journals.openedition.org/abpo/1742 ; DOI : 10.4000/abpo.1742 


\title{
Genre et pouvoir en cinéma Notorious (1946), Eyes Wide Shut (1999) ou de l'enchaînement des sentiments au déchaînement du désir
}

\author{
François ROUQUET \\ Maître de conférences en histoire contemporaine \\ CRAP/IHTP/Université Rennes 1/CNRS
}

Parmi les différentes approches possibles ouvertes par la très large question du genre et du pouvoir celle qui a été choisie ici s'appuie sur le double choix d'un lieu de pouvoir souvent négligé, celui du désir et des sentiments d'une part, de leurs représentations au cinéma d'autre part. Les premiers comme le second demeurent encore, quoiqu'une nette évolution soit perceptible depuis quelques années, parents pauvres des études historiques ${ }^{1}$. L'objet de cet article n'est pas en effet de s'attacher à historiciser la question des sentiments au cinéma, c'est-à-dire, selon l'acception la plus courante, l'étude chronologique d'une illustration des premiers par le second, que de souligner au contraire un effet de continuité, et de montrer pour le second vingtième siècle la grâce de l'art cinématographique dans la traduction des sentiments. De l'aprèsguerre des Enchaînés d'Alfred Hitchcock (1946) à la fin de siècle d'Eyes Wide Shut de Stanley Kubrick (1999), il s'agit moins de baliser un demi siècle de pouvoir sur le thème de l'amour au cinéma que d'évoquer, à travers ces deux exemples, l'écriture filmique des sentiments et du désir comme lieu de pouvoir sexué. Tout en restituant à l'œuvre de cinéma sa force singulière.

1. Cf. les présentations de Christian DELAGE et Nicolas RouSSELLIER du numéro spécial "Cinéma, le temps de l'histoire" (n46 d'avril-juin 1995) de Vingtième siècle. Revue d'histoire; et de Christian DELAGE et Antoine de BAECQUE de l'ouvrage collectif De l'histoire au cinéma, Bruxelles, Complexe-IHTP-CNRS, 1998. 


\section{Désir et cinéma}

Les études sur le genre, la différence de sexe ou les rapports sociaux de sexe sont rarement centrés sur les sentiments ${ }^{2}$. Si les rapports de genre sont désormais auscultés dans tous les recoins de la vie socio-politique, analyses de l'imagerie de la femme, approches sexuées en tout genre, et d'une façon générale, analyses fouillées des modalités de l'emprise de la domination masculine, etc., on s'intéresse assez peu en histoire à un lieu de pouvoir neutre (je ne parle évidemment pas des modèles qui en sont issus) où l'un et l'autre des deux sexes peuvent aléatoirement se trouver en position de domination. Je veux parler du domaine du désir et des sentiments, ou plutôt, puisque la part des uns et des autres n'est pas toujours facile à établir, du domaine des sentiments naissants. C'est-à-dire du moment de l'amour où chacun des protagonistes se trouve, souvent involontairement, parfois à son insu, dans une situation d'aliénation consentie, où, bien vite, le pouvoir de dépendance affective de l'autre, s'éveille à la conscience. La «science du genre», focalisée sur la question de la domination masculine tient à distance ce lieu d'expression d'un pouvoir indifférencié balisé en son temps par Roland Barthes dans son Fragment d'un discours amoureux ${ }^{3}$. Au cœur des relations entre hommes et femmes ${ }^{4}$, il s'agit d'un terrain trouble où une alchimie secrète régit l'ordre des plaisirs et des tourments, du pouvoir de la jouissance et de la souffrance. Lieu fascinant s'il en est, le monde des sentiments - trop souvent dévolu au féminin - demeure le terrain de jeu de la philosophie ou de la psychanalyse, plus rarement de l'histoire, presque jamais des gender studies, du moins quant à sa dimension essentielle ici

2. L'exemple des deux synthèses récentes publiées en France paraît significatif. Ainsi, la synthèse Histoire des femmes, dirigée par Georges DuBY et Michelle PERROT aux éditions Plon, dont le volume sur le $\mathrm{xx}^{\mathrm{e}}$ siècle sous la direction de Françoise THEBAUD (Plon, 1992, 647 p.) qui consacre des développements sur les fonctions et le statut dévolu aux femmes lors des guerres et sous les régimes totalitaires (patriarcat, fasciste, etc.), sur la société et leur place et les représentations dont elles sont l'objet; sur la maternité, la famille, l'État, le droit et la maternité, l'émancipation, le féminisme... L'article de Françoise CoLLIN, "La question des femmes en philosophie» approche cette question du désir mais à travers les théories philosophiques et la psychanalyse. Il en va de même pour l'Encyclopédie historique et politique des femmes (sous la direction de Christine Fauré), publiée aux PUF en 1997, dont le projet, conformément à son titre était de porter un regard "politique» sur la question. L'évacuation de la question du désir et des sentiments n'en demeure pas moins problématique, comme s'il s'agissait d'un lieu "à part" sans lien aucun avec l'histoire des femmes, qu'elle se revendique ou non comme politique.

3. Le Seuil, 1977. À l'article «Ravissement», on peut lire «Épisode réputé initial (mais il peut être reconstruit après coup) au cours duquel le sujet amoureux se trouve "ravi" (capturé et enchanté) par l'image de l'objet aimé (nom populaire: coup de foudre; nom savant: énamoration), p. 223.

4. Y compris l'homosexualité dans la mesure où les deux genres masculin et féminin sont, au moins pour ce qui est des rapports de pouvoir, réinvestis par les partenaires qui reproduisent, en l'adaptant, cette différence. 
c'est-à-dire cette attirance irrépressible soumise au diktat du désir ou à la fascination irrationnelle de l'autre imposée par l'amour. Ceci, en dehors des patterns et des modèles issus des sciences humaines attachées aux mécanismes conscients ou inconscients qui régissent l'économie des affects. Est-ce parce que l'amour est jugé trop conforme ou trop imprégné de l'imaginaire masculin dans les études féministes? Espace privilégié de la psychanalyse - dont les fondements théoriques procèdent également de cette même forme de domination - les lieux du désir semblent-ils inaccessibles aux historiens ${ }^{5}$ ? Outre que la question des sentiments demeure culturellement sous l'emprise de la vision classique des rapports de sexe inspirés du romantisme du XIX ${ }^{\mathrm{e}}$ siècle, le monde du désir et des sentiments désempare le regard de la science. Espace de l'intime et du secret, retors aux dogmes et aux révolutions fussent-elles féministes, posons ici que les lieux de l'amour - circonscrits à l'état amoureux et non à la déclinaison de ses effets sociaux inégalitaires - puissent être considérés, au moins à titre expérimental, comme un lieu de pouvoir. Un pouvoir au sens où l'état amoureux, en transformant notre rapport au monde et aux autres, nous inscrit dans un processus de dépendance réciproque qui confère à l'être aimé un pouvoir d'influence qui nous échappe. L'intérêt du domaine amoureux comme lieu de pouvoir tel qu'il est compris ici réside dans sa singularité de lieu neutre échappant à la domination masculine où chacun des deux sexes peut être dominant ou dominé. Parfois avec cruauté et sans qu'aucune règle morale, sociale ou politique puisse être de quelque recours dans l'analyse du phénomène. L'état amoureux invite à la jouissance comme à la souffrance, toujours dans un processus de consentement mutuel. Ce pouvoir de la domination, du charme exercé, de la possession, de l'ascendant sur l'autre, ou à l'inverse, cette jouissance de la dépendance consentie, se fondent dans une équation complexe au cœur de la nature humaine. Si les mécanismes de pouvoir dans le domaine des sentiments demeurent mystérieux, on tentera modestement ici de souligner l'usage de cette thématique telle que la comprend l'art cinématographique

Le second versant de cette approche justement, c'est qu'il est question de cinéma. Au cinéma, la conquête du pouvoir comme l'expression du désir sont des thèmes centraux. En cela, on peut dire que le cinéma est "habité» par le sujet. "Des relations entre hommes et femmes le cinéma a

5. Cf. entre autres, Philippe ARIES et Georges DuBY (sous la direction de), Histoire de la vie privée, 5 vol., Seuil, 1985-1987. L'exemple de deux livres publiés simultanément par Anne-Marie SoHN paraît significatif de la place de cet aspect de la question à l'articulation entre vie privée et étude de la sexualité: Chrysalides. Femmes dans la vie privée $X I X^{e}-X X^{e}$ siècle, Paris, Publications de la Sorbonne, 1996; Du premier baiser à l'alcôve. La sexualité des Français au quotidien (1850-1950), Paris, Aubier, 1996. Citons enfin l'approche historique sur un sujet voisin par Cécile DAUPHIN et Arlette FARGE (sous la direction de), Séduction et sociétés, Seuil, 2001. 
tout représenté, tout figuré, tout symbolisé», écrit Jacques Aumont ${ }^{6}$. Mais pas seulement parce que cette question du pouvoir et du désir est contenue dans la plupart des productions cinématographiques comme elle est le ressort de la plupart des vies humaines. "Ce qu'il y a de plus spécifique dans les moyens du cinéma, c'est de toute évidence le pouvoir de concrétiser les puissances de l'amour" pouvait-on lire dans L'Âge du cinéma en $1951{ }^{7}$. Sans doute aussi parce que cette projection du désir et de la puissance se décline également dans le rapport que le spectateur entretient avec ce qu'il voit sur l'écran. L'aphorisme célèbre de Bazin, "le cinéma substitue à notre regard un monde qui s'accorde à nos désirs" demanderait à lui seul un long développement sur son incidence dans la relation qu'entretient l'histoire avec le cinéma. Lieu de passion et de jugement, les films cristallisent le débat social, privé ou public, parce que chacun y projette son propre désir d'identification, de fiction et de résolution de ses propres passions intérieures

Le cinéma, art de masse, est un dispositif technologique qui s'est de plus en plus sophistiqué au cours du siècle en construisant un univers imaginaire ayant sa propre puissance. Cet univers est à la fois produit du projet commun d'un groupe de créateurs (scénariste, réalisateur, acteur, monteur, ...) et d'un pari économique (producteur, distributeur, exploitants). En cela un film est un objet complexe, conjonction entre des aspirations créatives plurielles et les enjeux économiques de son temps. Le cinéma peut être représentatif de son époque ou au contraire contradicteur de "l'imaginaire dominant" ou des "représentations dominantes». Mais comme toute œuvre d'art, un film se crée un espace temporel d'autonomie. Car sa qualité d'œuvre lui fait traverser le temps chronologique, comme Gilles Deleuze l'a montré ${ }^{8}$ en s'appuyant sur Bergson ("le passé coexiste avec le présent qu'il a été»), et saint Augustin («il y a un présent du futur, un présent du présent, un présent du passé»). À l'évidence, cette dimension pose problème dans la mise en place d'un protocole d'analyse qui soit opératoire en histoire, mais du moins doit-on en rappeler l'existence et toujours considérer la complexité de l'objet filmique dès lors que l'on prétend l'utiliser comme source. Le cinéma ne peut être réduit à une simple imagerie produit d'une chronologie com-

6. Qui ajoute: «Il n'est pas un seul type de relation des sexes humains qui n'ait donné un modèle de scénario, de personnage, de récit. La combinatoire des âges, celle des situations; la gamme des affects; les couples possibles et impossibles; légitimes et horsla-loi; ceux qui sont destinés à durer et ceux qui vont s'épuiser, tout cela a trouvé ses équivalents et ses incarnations dans l'art du film", "Man meets girl", in AUMONT, Jacques (sous la direction de) La différence des sexes est-elle visible? Les hommes et les femmes au cinéma, Cinémathèque française, 2000, p. 393.

7. "Comme dans un bois» in L'Âge du cinéma, août-novembre 1951. Cité par VIRMAUX, Alain et Odette, Dictionnaire du cinéma mondial, Éditions du Rocher, 1994.

8. DeleuZe, Gilles, Cinéma 1. L’image-mouvement, Éd. de Minuit, 1983; Cinéma 2. L'image-temps, éd. de Minuit, 1985; et aussi du même avec GUATTARI, Félix, Mille plateaux, Éd. de Minuit, 1980. 
mode mais réductrice, alors que le propre même d'un film est de jouer avec "le temps compté». La fiction est à la fois représentation de son temps et hors du temps. Cette proposition, si on l'accepte, induit l'idée d'une spécificité du cinéma et à travers lui, l'ambiguïté de notre rapport au temps. Une spécificité de contenu associée à l'existence de toute œuvre d'art ${ }^{9}$, mais aussi une spécificité liée au support lui-même, à sa capacité de projection collective (et longtemps nationale), à son extraordinaire pouvoir d'identification et d'investissement de l'imaginaire, à sa redoutable efficacité d'imprégnation des esprits. Ce que Henri Agel appelait "l'art de la célébration ${ }^{10}$ " amène nécessairement à s'interroger sur la dimension plurielle et complexe de ce que dit le cinéma à l'historien ${ }^{11}$. Ainsi la question du genre et du pouvoir posée sous l'angle des sentiments entretient-elle un étrange effet de miroir avec le matériau filmique choisi ici pour l'ausculter. C'est que l'une comme l'autre obligent à une certaine prise de risque: moins dans l'approche de l'analyse filmique que dans la certitude d'évacuer toute idée d'élucidation qui se voudrait définitive face à un sujet volatile et mystérieux et, qui plus est, dans l'étude de ses représentations ${ }^{12}$. Nous nous trouvons donc face à une double mise en intrigue. La première est celle des sentiments qui, dans le rapport amoureux, s'érigent sur la mise en fiction de l'être aimé. La seconde est celle du cinéma dont la fiction est l'essence même. L'une représentée par l'autre multiplie ainsi les effets de miroir.

\section{Des films en miroir}

Sentiments et fiction. Si la dimension littéraire du sujet ne fait aucun doute, si elle lui confère même un surcroît d'intérêt dans ce qu'il est convenu aujourd'hui d'appeler "l'histoire culturelle», sa double fluidité impose, sauf à risquer un discours circulaire qui n'aurait d'objet que de se

9. «Loin de constituer un simple catalogue d'informations sur ce qui s'est passé, le cinéma construit avec ses spectateurs une relation esthétique et historique (...), nous aidant à mieux comprendre la corrélation entre "l'intériorité de notre mémoire" et "le processus de notre socialisation", entre une représentation du monde (le cinéma) et la manière dont les hommes vivent dans ce monde (l'histoire)" écrivent, citant Paul RIC UR, Antoine DEBAECQUE et Christian DELAGE, dans l'introduction de l'ouvrage collectif De l'histoire au cinéma, Bruxelles, Éd. Complexe-IHTP, 1998.

10. AGEL, Henri, Un art de la célébration: le cinéma de Flaherty à Rouch, $7^{\mathrm{e}}$ Art, 1987.

11. On retiendra parmi d'autres outre les "classiques" de Marc FERRO et celle citée précédemment, le numéro de la revue Vertigo "Le cinéma face à l'histoire", n ${ }^{\circ}$ 16, 1997; ou celui de Vingtième siècle. Revue d'histoire, "Cinéma et histoire", n46, 1995; et pour une approche bibliographique globale, l'étude de LINDEPERG, Sylvie, Clio de 5 à 7 . Les actualités filmées de la Libération: archives du futur, CNRS Éditions, 2000.

12. Signalons la récente publication sous la direction de AUMONT, Jacques, La différence des sexes est-elle visible? Les hommes et les femmes au cinéma, Cinémathèque française, 2000. La critique cinématographique anglo-saxonne dans le domaine du genre est, on le sait beaucoup plus développée. Pour une première approche, cf. les textes présentés par BuRch, Noël, dans Revoir Hollywood. La nouvelle critique anglo-américaine, Nathan-Université, 1993. 
satisfaire lui-même, de rappeler, quoique la question demanderait de longs développements théoriques, quelques principes fondamentaux qui régissent l'analyse d'un film ${ }^{13}$. Paradoxalement, c'est à partir d'une réflexion menée par Hélène Puiseux sur les actualités allemandes, sujet de sa thèse en 1978, qu'il peut être utile de mettre en perspective un regard possible sur la fiction et sur le cinéma en général puisque, nous le savons, quelque forme qu'il prenne, le cinéma est une fiction. Comme la fiction amoureuse célèbre les relations entre hommes et femmes, le cinéma projette à la face du monde un autre monde qui nous aide à vivre. C'est dans cette perspective qu'Hélène Puiseux soumettait à trois principes le fondement de l'analyse des films.

1- Du point de vue théorique, l'analyse de ces documents s'appuie sur les travaux de référence en sciences humaines, de Georges Dumézil ${ }^{14}$ à Claude Lévi-Strauss ${ }^{15}$, Jean-Pierre Vernant ${ }^{16}$ ou bien d'autres. Ces auteurs ont montré que les grands ensembles de récits, quelle que soit leur forme (épopée, tragédie, contes, etc.) avaient une fonction commune qui était celle de penser le monde. "Ces récits, écrit Hélène Puiseux en s'inspirant des travaux de Greimas, exposent et se chargent de rendre admissible (si inadmissible soit-elle), l'armature du monde où ils naissent et se répandent selon un mode d'appropriation collective ${ }^{17}$ ".

2- On peut appliquer cette définition du mythe au cinéma, c'est-à-dire poser que le cinéma est le mode d'expression actuel des mythes (anciens ou modernes); que comme les mythes anciens expliquent le monde ou aident à supporter le monde, les films (ou d'une certaine manière, la télévision) médiatisent le quotidien de l'individu et l'aident à supporter l'existence. C'est-à-dire que cette médiatisation nous aide à supporter au moins trois problèmes fondamentaux qui sont autant de sources de frustrations et d'angoisse: le premier est la vie, donc la dégénérescence et la mort; le second, le sexe et la sexualité, c'est-à-dire la pulsion et/ou la frustration sexuelle; le troisième, la socialisation: supporter les contraintes de la vie avec les autres. "En racontant les frottements qui naissent entre la société [...] et les individus, explique Hélène Puiseux, [les mythes] proposent des modèles pour résoudre, juger ou dépasser ces frottements qui constituent le matériau de chaque récit particulier, qui sont autant

13. Étant entendu que les approches en ce domaine peuvent être variées et sujet d'un débat sans fin sur ce que dit un film.

14. Mythe et épopée (I, II et III, Gallimard, 1968,1971,1978) où l'auteur cherche, à partir des faits de langue et de faits de culture, le prototype commun d'une civilisation (indo-européenne).

15. Mythologiques I,II,III et IV (Gallimard, 1968-1975) où l'apôtre principal du structuralisme a montré, à partir d'environ un millier de contes et récits d'Indiens d'Amérique du nord et du sud, les points de jonction originels possibles dans l'analyse des mythes.

16. Mythe et pensée chez les Grecs, Gallimard, 1965, qui a effectué la même démarche à propos des mythes grecs.

17. PuiseuX, Hélène, «Du rite au mythe: les actualités», Ciném'Action $n^{\circ} 65,4^{\mathrm{e}}$ trimestre 1992, pp. 96-104. 
d'infractions à la norme et par là même contribuent à la fixer par défaut ${ }^{18}$ ». Et c'est bien de cette norme qu'il s'agit, qu'elle soit sociale (mariage, éducation, rituels divers), juridique (la loi), mais aussi sensorielle ou culturelle ou idéologique (l'espace et le temps, les systèmes de pensée et les représentations du monde).

3- Donc à partir de ces présupposés, les films doivent être perçus comme concentrant les lignes de force d'une société, lignes de force qui se révèlent dans une approche plurielle:

- le monde interne du film, dont la genèse montre qu'il est le produit complexe de choix esthétiques et socio-économiques, mais aussi objet de contraintes idéologiques et parfois partiellement fruit du hasard;

- le monde externe (la société d'où il émerge et qui définit ses conditions de production mais aussi qui lui fournit ses référents);

- la place qu'y occupe l'œuvre, à la fois pôle de valeurs culturelles au cours d'une période donnée et agent permanent d'un questionnement collectif sur son contenu. On pourrait ajouter pour les films plaçant explicitement le passé au centre de leur discours, le monde présent, c'est-à-dire notre propre demande permanente de renouveau du passé. Les films à vocation "historique» de ce point de vue proposent une écriture filmique de l'histoire qui n'est pas toujours considérée en tant que telle par les historiens, souvent cantonnés dans la seule appréciation de la concordance d'un film avec l'époque de sa production.

L'œuvre filmique n'est donc pas un objet simple. Si comme d'autres sources, elle nous renvoie à une réinterprétation du passé, elle a pour elle de privilégier dans ce processus de réinterprétation notre position de spectateur et notre rapport au temps. Le premier fait de nous moins des citoyens en demande d'histoire que des spectateurs avides d'une esthétique de l'histoire sans cesse en devenir. Le second est à la fois un lieu et un jeu de brouillage permanent qui multiplie les effets de miroir entre la construction (ou la déconstruction) du temps telle qu'elle s'opère en cinéma et le temps construit du spectateur-Sujet. À cette stratification du sens et du temps, s'ajoute celle du désir et des sentiments qui émanent du cinéma et qui projettent le spectateur dans un double univers intime et collectif où le pouvoir appartient aux images. C'est là l'intérêt du film de Stanley Kubrick, Eyes Wide Shut, qui joue sur le désir et son impossibilité en opposant l'image, produit de l'imagination individuelle et acte de liberté, à l'illustration, produit aliénant d'un modèle esthétique dominant ${ }^{19}$. Le film, ici mêlé à son objet, est donc à concevoir, selon la formule

18. Ibidem.

19. "L'image est une projection mentale. L'illustration est en revanche ce qu'il y a de plus contraire à l'imagination. Eyes Wide Shut est l'histoire d'un être singulièrement dépourvu d'imagination, qui espère accéder au monde d'interdits alors qu'il se soumet à une esthétique dominante, qui influence ses plus profond fantasmes.» SAADA, Nicolas, «Scènes de l'envie conjugale», Cahiers du Cinéma, n 538 , p. 33. 
de Jean-Luc Godard, et en dépit des difficultés que cela entraîne, comme une «forme qui pense».

Ces considérations ont une incidence sur le choix des deux films évoqués ici dans cette perspective thématique du genre et du pouvoir. Essentiellement par le fait que leur choix, outre sa part d'arbitraire, se justifie par cette forme d'autonomie émanant d'un film, par sa qualité d'œuvre et cette dimension intemporelle qui lui est liée. J'avais initialement construit un petit corpus de films balisant le second vingtième siècle, choisis à partir d'un critère double: il fallait qu'ils proposent une représentation explicite de la question du genre et du pouvoir dans le domaine des sentiments et du désir; il fallait ensuite que leur qualité d'œuvre, c'est-à-dire leur capacité à perdurer, à demeurer en phase ou à résonner dans l'imaginaire public ou critique, soit reconnue. Bref des films dont les qualités intrinsèques réactualisent en traversant le temps la question qui nous occupe ici. Pour des raisons pratiques - l'étude d'un tel corpus ferait l'objet d'un ouvrage entier - il n'en subsiste ici que deux qui illustrent la question sous deux angles complémentaires. Le premier est Notorious (Alfred Hitchcock, 1946), le second Eyes wide shut (Stanley Kubrick, 1999). Le premier est centré sur les sentiments dans le jeu de pouvoir permanent entre un homme et une femme au sein d'un scénario d'espionnage, tandis que le second raconte la découverte par un couple de son aveuglement et de la puissance inconsciente du désir lorsqu'il fait irruption dans les certitudes sentimentales bourgeoises. Ces films qui bornent chronologiquement le second vingtième siècle proposent deux modes de représentation du pouvoir dans le rapport amoureux. Et si ils reflètent leur époque et leur contexte, l'évolution des mœurs, des usages et des rapports sociaux en général, ils attestent également de la profondeur et de l'intemporalité d'une thématique toujours rejouée entre homme et femme, comme au cinéma.

\section{Les enchaînés (Alfred Hitchcock, 1946) ${ }^{20}$ : les sentiments ou la névrose et la rédemption}

Initié par David Selznick en 1944 qui ne disposait à cette époque que de la moitié d'un scénario écrit par Ben Hecht (tiré d'un texte Song of a dragon publié dans la presse), d'Alfred Hitchcock et d'Ingrid Bergman sous contrat, le projet de Notorious fut ensuite revendu à la RKO par le producteur qui s'en désintéressa ${ }^{21}$. Le premier traitement du scénario fut achevé en janvier 1945, tourné entre octobre 1945 et janvier 1946. Le film sortit en juillet 1946. Soulignons que la genèse de Notorious pourrait être

20. Scénario de Ben Hecht d'après un sujet d'Alfred Hitchcock (Song of the dragon); photo, Ted Tezlaff; musique, Roy Webb; interprétation, Ingrid Bergman, Cary Grant, Claude Rains, Louis Calhern, Leopoldine Konstantin.

21. Ce qu'il regretta amèrement par la suite puisque le film gagna huit fois son budget. 
un bon exemple de la part de hasard pouvant susciter une œuvre d'art. Ce film mal né, revendu en cours d'ébauche et écrit pour moitié en cours de tournage - ainsi cinq fins différentes furent envisagées avant de donner lieu à la magistrale scène de l'escalier -, cumulait les handicaps et bégaiements avant de trouver son plein épanouissement d'œuvre. Si le cas n'est pas exceptionnel, du moins atteste-t-il à sa manière de la puissance et de l'autonomie de l'œuvre d'art envers et contre tout.

Notorious raconte l'histoire d'une jeune femme, dont le père, après la Seconde Guerre mondiale, a été condamné pour espionnage au profit d'anciens nazis continuant la lutte. Il se suicide peu de temps après son procès. Privée de son père Alicia Huberman (Ingrid Bergman) mène une vie dissolue, entre beuveries et amants de passage. Elle rencontre un jour Devlin (Cary Grant) dont elle tombe amoureuse. Mais Devlin est un dur à cuire, très méfiant avec les choses de l'amour et de surcroît agent de la CIA envoyé pour convaincre Alicia d'accepter une mission au Brésil. Il s'agit de reprendre contact avec un ami de son père, Alexander Sebastian soupçonné de préparer un dangereux complot avec des nazis continuant la lutte. Alicia accepte par amour pour prouver à Devlin (et à elle-même) qu'elle n'est pas la femme perdue qu'il voit en elle. Devlin apprend qu'Alicia doit séduire Sebastian afin d'infiltrer le réseau. Il espère qu'elle va refuser par amour pour lui. Alicia espère que Devlin va l'en empêcher par amour pour elle. Dans cette crise de confiance mutuelle, l'un comme l'autre joue le rôle qui lui est assigné. Alicia séduit Sebastian (personnage vivant sous la coupe de sa mère), livre les noms de ses complices à Devlin lors de rendez-vous secrets ou chacun reproche à l'autre son attitude. Sebastian demande à Alicia de l'épouser. La CIA n'en espérait pas tant, mais face à l'indifférence (feinte) de Devlin, Alicia accepte. Mariée, elle dispose de toutes les clés de la maison sauf une, celle de la cave jalousement conservée par son mari. Devlin lui demande d'organiser une soirée au cours de laquelle il entre dans la cave avec Alicia qui a pu subtiliser la clé. Tous deux percent le secret des nazis: les bouteilles de la cave sont remplies de minerai d'uranium. Surpris par Sebastian, ils s'embrassent pour préserver leur découverte. Sebastian comprend qu'il est en danger et prévient sa mère qu'il a épousé un agent américain. Celle-ci décide d'empoisonner lentement Alicia laquelle apparaît un peu chancelante au rendez-vous hebdomadaire avec Devlin qui la croit replongée dans l'alcoolisme. Mais l'absence d'Alicia au rendez-vous suivant éveille ses soupçons. Devlin se rend chez Sebastian et sauve Alicia sous les yeux des nazis au cours d'une scène d'anthologie.

Le titre original (Notorious: une femme de mauvaise réputation) comme sa traduction française, Les enchaînés, soulignent deux versants du film au cœur de la problématique "genre et pouvoir" dans le rapport amoureux. Il s'agit tout d'abord d'un modèle ou plutôt d'un contre-modèle, celui de la femme en dérive, belle, alcoolique, aventurière; qui s'oppose au sortir de la guerre à celui de la ménagère, mère de famille, pilier de la nation de l'American way of life. C'est sa déviance qui donne à Alicia la 
force de lutter à la fois contre et pour l'ordre politique masculin. Il s'agit ensuite d'un "enchaînement» collectif des personnages et surtout naturellement des deux personnages principaux:

- Devlin prisonnier de sa fonction et de son aveuglement se défie de l'amour pourtant sincère d'Alicia; sincérité qui se révélera à lui dans la scène finale. Il est le relais de la manipulation de la CIA. Il est le fossoyeur de son amour refoulé. Il est aussi l'orchestrateur de la rédemption d'Alicia jusqu'au moment ultime de la mort programmée de celle-ci, où l'amour se révèle à lui;

- Alicia est la figure de la jeune femme américaine sans homme (privée de père et de mari), donc perdue. Elle n'a de cesse de prouver qu'elle peut être digne de l'homme qu'elle aime.

Devlin et Alicia jouent un jeu intime qui reproduit la subtilité tactique de la partie d'espionnage qui se joue. Se construit ainsi le double jeu entre l'intérêt de deux êtres et deux intérêts politiques. Un emboîtement du jeu de l'amour et de la politique où les rapports de force sont en permanence au centre du film.

- Les membres de la CIA sont les grands manipulateurs de Devlin et d'Alicia au nom de la logique d'État et de la sauvegarde de l'intérêt supérieur de la nation;

- Alexander Sebastian est la figure masculine sous une triple domination: celle de sa mère, avec laquelle il vit et dont il ne peut se détacher; celle du réseau nazi dont les membres lui font peur, celle enfin d'Alicia dont il ne perçoit le jeu que lorsqu'il est trop tard. Personnage masculin a priori négatif parce qu'il est celui qui veut sa mort, Alexander Sebastian est malgré tout l'homme qui aime le plus Alicia. La mère plus masculine que son fils est une femme de tête, calculatrice, possessive et dominante. Lui n'est qu'un pauvre petit mâle n'ayant jamais pu s'émanciper de cette maîtresse-mère dont le visage rayonne lorsque piteux, il vient lui demander de l'aide. C'est elle qui décide immédiatement du meurtre lent d'Alicia;

- enfin, les membres du réseau nazi sont enchaînés par leur projet fou et par la terreur qu'ils font régner au sein de l'organisation ${ }^{22}$.

Cette opposition entre les deux personnages masculins fut voulue par le scénariste Ben Hecht, féru de psychanalyse, pour qui "les hommes aiment chez les femmes leur propre féminité perdue». Bill Krohn établit le lien entre le personnage de Devlin et ce que Hecht, obsédé par l'Holocauste, avait écrit dans une étude qu'il avait précédemment rédigée sur l'antisémitisme (A Guide for the Bedevilled). «Devlin lutte contre l'attraction exercée par la féminité d'Alicia, écrivait Hecht dans cet ouvrage, tout comme l'antisémite lutte avec le Juif comme un singe attaquerait sa propre

22. C'est ainsi qu'au cours d'un dîner, Émil, le maître d'hôtel, ayant laissé entrevoir son trouble parce qu'il avait cru que les bouteilles servies étaient celles contenant de l'uranium est physiquement éliminé. 
déplaisante image dans le miroir ${ }^{23} "$. Face à lui, Sebastian Alexander serait celui qui, façonnant son amour dans la délectation, "plonge dans sa féminité perdue».

Les sentiments et le pouvoir de l'autre sexe constituent la matrice des Enchaînés ${ }^{24}$. Ils ne sont pas seulement le ressort de l'œuvre. Leur expression porte la trace d'une censure qui ne badinait pas avec le cinéma, art dont la capacité d'influence sur le public était jugée trop puissante pour ne pas être étroitement contrôlé ${ }^{25}$. C'est ainsi qu'une scène fut supprimée où Devlin exprimait à Prescott son souhait d'être muté, et où il sousentendait qu'il s'était mis à boire pour endurer sa souffrance morale ${ }^{26}$. La légèreté avec laquelle Prescott abordait le divorce possible d'Alicia après sa mission parut déplacée. On relèvera cependant l'ambivalence de situation sur laquelle joue le film en mettant en scène, pour défendre l'Amérique et ses valeurs, une jeune femme américaine qui sacrifie justement les valeurs du mariage et de la famille sur l'autel de l'intérêt d'État. L'intérêt national s'ajuste aux raisons personnelles et affectives d'Alicia. Celui de la CIA s'accorde à la rédemption de la Notorious qui prend toute sa valeur précisément parce qu'Alicia rejoue pour l'intérêt suprême de l'État sa vie (son péché) antérieure, alors qu'elle est amoureuse de "son ange gardien". cette figure féminine christique d'Alicia est du reste célébrée dans la scène finale ou elle attend la mort tandis que Devlin, prince charmant (névrosé) vient enfin la sauver.

Le film est concentré sur la force des sentiments même si c'est au fond la contrainte qui les révèle: dévouement patriotique et sens du devoir (par dépit) de Devlin et d'Alicia, loyauté, rachat des fautes commises, mais aussi jeu de pouvoir: domination, soumission, manipulation et jeu de dupe, ambivalence du courage dans un paradigme politico-sentimental où la morale s'inverse. Le genre de l'espionnage s'accorde parfaitement

23. KROHN, Bill, Hitchcock au travail, Éd. Cahiers du cinéma, 2000, p. 101.

24. Cf. infra, l'extrait de la scène entre Devlin et Alicia. On remarquera dans ce même dialogue la manière dont Alicia joue la mère avec Devlin pour le faire parler ("Raconte tes malheurs à maman ou le poulet ne passera pas. Allons Monsieur Mystère...")

25. Par le célèbre Motion Picture Production Code (MPPC), plus connu sous le nom de Code Hayes (du nom de son initiateur, William $\mathrm{H}$. Hayes) qui réglementa les interdits entre 1930 et 1966; mais aussi et surtout par l'autocensure que pratiquaient les Majors elles-mêmes.

26. "Devlin: Avec chaque verre de plus, j'ai envie d'aller chez Sebastian et de le liquider. Allez-y, riez!

Prescott: Je ne ris pas

Devlin: Pourquoi pas? Ce genre de plaisanterie n'est pas monnaie courante !

Prescott: Cela ne peut guère durer plus longtemps. Elle en aura bientôt fini avec cette mission. (avec un brin d'humour). Peut-être pourrez-vous ensuite aider les Sebastian à obtenir un divorce bien ficelé.

Devlin: À quoi bon? Elle en a fini avec moi, de toute façon. Et je ne peux pas lui en vouloir. Je n'ai pas cessé de l'agresser depuis que nous sommes ici. Et maintenant je me retrouve avec ce noud à l'estomac que je ne peux plus supporter...”, cité in Bill KROHN, Hitchcock au travail, Éd. Cahiers du Cinéma, 1999, p. 92. 
de cette mise en miroir entre les deux stratégies du pouvoir et de l'amour et la mise en œuvre de leur subtile mécanique. Quoiqu'il puisse être difficile de dissocier les sentiments du désir sans entamer un débat aventureux dans le domaine de la psychanalyse, on peut considérer que le désir s'efface ici devant les sentiments. On comprend en effet facilement qu'au cours de leur séjour au Brésil, Devlin et Alicia ont consommé leur désir réciproque ${ }^{27}$. C'est d'ailleurs tandis qu'elle joue à l'épouse-ménagère entourant son (faux) mari de toutes ses attentions qu'Alicia, se trouve brutalement rejetée de la norme qu'elle tentait vaillamment d'intégrer par amour pour lui ${ }^{28}$. Cette inversion du processus amoureux participe de cette idée que, tout entre les deux personnages commence, non par le désir, mais par la naissance d'un amour où le désir n'a été qu'un instrument du pouvoir. Dans Notorious, le désir est instrumentalisé par le pouvoir d'État manipulateur des deux héros. Devlin doit séduire Alicia pour qu'elle prenne contact ensuite avec Sebastian (et qu'elle le séduise). Le désir dépourvu de sentiment est également ce qui caractérise l'environnement de dépravation d'Alicia avant qu'elle ne rencontre Devlin. C'est donc cette figure d'inversion du jeu désir-sentiment qui place le second au centre de la mise en intrigue du film, et c'est pour finir, la force de ce renversement qui rétablit, en même temps que la happy end, l'ordre moral puritain.

Plusieurs éléments donnent au film une dimension particulière qui l'inscrivent dans le contexte d'une guerre à peine achevée et renseignent sur les intentions des auteurs (Ben Hecht et Alfred Hitchcock). Tout d'abord, les nazis qui, en 1946, ne sont pas un simple élément pittoresque, de même que la compromission de IG Farben évoquée dans le film. L'après-guerre marque de son ombre le scénario de ce film d'espionnage. L'Espagne évoquée à plusieurs reprises est la tête de pont permettant la fuite des nazis vers l'Amérique du sud. Les réseaux nazis se déployaient à travers le monde, qu'il s'agisse d'organiser la fuite et l'exil des partisans d'Hitler mais aussi de poursuivre la chimère du Grand Reich. L'intérêt porté par les deux auteurs du film à la lutte contre le nazisme va également dans ce sens. Le scénario tient autant par la relation entre les deux personnages principaux que, en 1946, par la crédibilité de son contexte. On sait en effet que l'Holocauste obséda Hecht au cours de la phase d'écriture. Alfred Hitchcock lui-même répondit favorablement à la demande britannique de supervision du montage de films sur la libération de dix camps de concentration, notamment ceux de Belsen et d'Auschwitz ${ }^{29}$. Enfin, l'idée du minerai d'uranium et de la bombe ato-

27. Le passage du voussoiement au tutoiement entre Devlin et Alicia dès leur arrivée au Brésil ne laisse aucun doute sur ce point.

28. Cf. infra, l'extrait cité.

29. Face à la nouvelle donne politique qu'imposait la guerre froide, ce film, Memory of the camps ne fut guère apprécié du gouvernement et ne fut finalement projeté qu'en 1984 aux États-Unis. 
mique, dont personne à peu près ne connaissait l'existence au moment où le film est écrit puis tourné, constitue un point de concordance entre l'imagination des créatifs et la technologie militaire qui surprit jusqu'à certains hauts responsables du ministère de la défense ${ }^{30}$.

Manière de Traviata d'après guerre, le film Les Enchaînés présente un aspect de la question du genre et du pouvoir: le tragique de situation dans l'entrelacs de l'intime et de l'intérêt public, ou la leçon de morale est dispensée par la déviante, la Notorious. Alicia est l'objet d'une manipulation machiavélique des gardiens de l'intérêt d'État. Elle est à la fois victime et maîtresse d'un destin que les circonstances lui permettent justement (et paradoxalement) de reprendre en main. Elle est à la fois femme manipulée, pion de la domination masculine et pièce maîtresse du dispositif mis en place. Sur elle repose la réussite de l'opération, et c'est l'héroïne lucide et désabusée qui donne le change à la pression masculine. L'acceptation de son sort d'espionne la place sur une ligne de partage entre deux hommes, celui qu'elle aime et celui dont elle abuse. Le premier exerce malgré lui un pouvoir dicté par sa fonction, et sans doute aussi par une forme de perversité et de refus d'accepter l'amour qu'elle lui propose; le second lui offre tout et, aveugle, subit la domination d'Alicia. Mais c'est bien de sentiment qu'il s'agit ici. En cela, le film Les Enchâ̂nés est une œuvre qui cultive l'équivoque sur la réalité du pouvoir individuel des genres.

\section{Extrait}

Devlin et Alicia arrivés au Brésil sont installés dans un appartement. Alicia décidée à devenir une épouse pour celui qu'elle aime décide de se mettre à faire la cuisine pour lui, ce qu'elle n'a jamais ni su ni essayé de faire pour un homme. Tandis qu'elle prépare le repas, Devlin est appelé par Prescott. Alicia lui demande de rapporter du champagne. Prescott apprend à Devlin qu'Alicia doit séduire Alexander Sebastian. Il sort du bureau en oubliant le champagne.

Fondu enchaîné de la bouteille à l'appartement.

Intérieur/nuit

(La caméra suit Devlin qui traverse l'appartement en plan américain de droite à gauche)

Alicia: Le poulet a pris plus de temps que prévu. Qu'ont-ils dit? (plan américain sur Alicia dans la cuisine, légère contre-plongée). J'espère qu'il n'est pas trop cuit. Une fois j'y ai mis le feu. (plan moyen large de Devlin de dos qui marche dans le salon qui sort sur le balcon puis penche la tête en signe d'abattement) Il vaut mieux le couper dans la cuisine. A

30. Cf. KROHN, Bill, op. cit. qui explique qu'Alfred Hitchcock, s'étant renseigné sur la possibilité fission de l'uranium auprès d'un physicien (afin de tester la crédibilité de son scénario) fut un temps surveillé par les services de contre-espionnage américains. Cf. également la bibliographie exhaustive établie par l'auteur dans ce même ouvrage. 
moins que vous ne vouliez un demi-poulet. Nous aurons des couteaux et des fourchettes finalement. (plan américain d'Alicia qui sort de la cuisine avec le poulet, la caméra la suit, Alicia rejoint Devlin et recadre comme dans le plan précédent) S'occuper d'un mari tous les jours doit être merveilleux.(elle l'embrasse en passant) Il fait peut-être trop frais (plan rapproché, Alicia enlace Devlin) et l'embrasse plusieurs fois) reprenons où nous en étions? (face à la mine fermée de Devlin) Qu'est-ce qu'il y a? Des ennuis? Raconte tes malheurs à Maman, ou le poulet ne passera pas. Allons, Monsieur Mystère, qu'y a-t-il?

Devlin: Après le dîner.

Alicia: Maintenant. (silence Devlin). Je vais t'aider. Tu as une femme et deux adorables bambins. Notre histoire ne peut pas durer.

Devlin: Tu as souvent dû l'entendre.

Alicia: (gros plan d'Alicia, angle interne, son visage se ferme)... Toujours des coups bas !... Tu es injuste.

Devlin: (contre champ, gros plan sur lui, angle interne) Oublie ça. Ne pensons qu'au travail qui nous attend

Alicia: (gros plan) ce travail existe donc !

Devlin: (gros plan) Te rappelles-tu un certain Sebastian?

Alicia: (gros plan) Alex Sebastian... C'était un ami de mon père.

Devlin: (gros plan) Il en pinçait pour toi

Alicia: (gros plan) en pure perte (gros plan de Devlin)

Devlin: Il est ici à la tête d'une grosse firme (gros plan d'Alicia)

Alicia: Sa famille a toujours eu de l'argent.

Devlin: C'est un nazi pour qui la guerre continue.

Alicia: quelque chose d'important (gros plan de Devlin)

Devlin: oui sur une grande échelle. Il faut le contacter (gros plan d'Alicia qui comprend)

(plan rapproché de Devlin et Alicia. Elle s'éloigne de lui et s'assoit sur le balcon, le cadre s'élargit)

Alicia: Courage. Continue

Devlin: Nous le rencontrerons demain. Le reste te regarde. Mais accrochele (plan rapproché d'Alicia de face)

Alicia: Mata-Hari. Elle fait l'amour contre des dossiers.

Devlin: il n'y a pas de dossiers. (il vient se placer derrière elle à distance) Découvre ce qui se trame chez lui et au sein du groupe.

Alicia: Tu as toujours su en quoi consistait ma mission? (Devlin en gros plan)

Devlin: Je viens de l'apprendre

Alicia: (gros plan) Ne leur as-tu pas dit... que je n'étais pas ce genre de fille? (plan épaule de Devlin, légère contre-plongée)

Devlin: J'ai pensé: "elle peut toujours refuser»

Alicia: (gros plan) Et tu as dit que dans huit jours Sebastian serait à mes pieds

Devlin (plan épaule): Je n'ai rien dit (gros plan d'Alicia)

Alicia: Pas un mot pour une femme qui t'aime?

Devlin (plan épaule en contre plan): Les ordres sont les ordres (Les deux de face, Devlin debout en plan américain, Alicia au premier plan assise)

Alicia: Ne te fâche pas Dev. Je fais ma crise de romantisme. J'espérais un cri du coeur. "Comment osez-vous soumettre la pure Alicia, la nouvelle Alicia à ce destin cruel? (elle se lève et s'approche de lui) 
Devlin: ça ne me fait pas rire (même plan, les deux de face en plan rapproché)

Alicia: Tu veux que j'accepte?

Devlin: A toi de voir

Alicia: Je te pose une question

Devlin: A toi de décider

Alicia: tu restes de marbre (gros plan d'Alicia de face) Dis-moi ce que tu ne leur as pas dit, que je suis respectable, que je t'aime.(gros plan de Devlin, angle interne)

Devlin: J'attends ta réponse (plan rapproché des deux)

Alicia: Ce que c'est qu'un ami ! (la caméra la suit tandis qu'elle avance vers la droite) pas la moindre confiance en moi hein? Qu'Alicia retourne dans son élément: le caniveau... Oh Dev !... Dev... (on la voit à travers la vitre de la cuisine se servir un verre qu'elle avale d'un coup) Je commence quand?

Devlin: Demain matin. (plan rapproché, il avance jusque vers Alicia, tous les deux sont dans le cadre)

(plan de la table dressée pour le dîner)

Alicia: Quelle mauvaise idée, (retour au cadre précédent) tout a refroidi. Que cherches-tu?

Devlin: J'ai du laisser le champagne en route.

Fondu au noir.

\section{Eyes wide shut (Stanley Kubrick, 1999) ${ }^{31}$ ou le déchaînement vain du désir}

Si le désir demeure elliptiquement traité dans Notorious, c'est sans doute autant par puritanisme que par nécessité dramatique puisqu'il est, dès le début du film, consommé sans amour et associé à la déchéance d'Alicia. Kubrick sur ce même thème propose un demi-siècle plus tard une autre variation dans le registre des genres et du pouvoir, centrée cette fois sur la dimension irrépressible du désir et sa tentative toujours vaine de satisfaction.

Adapté du texte Traumnovelle (La nouvelle rêvée, ou Rien qu'un rêve selon les traductions) qu'Arthur Schnitzler ${ }^{32}$, Viennois, médecin, ami de Freud et fasciné par l'interprétation des rêves, publia en 1926 après 17 ans de gestation. Comme d'autres films de Kubrick, le projet Eyes wide shut fut également long à aboutir. Trente ans s'écoulèrent entre le moment où le réalisateur songea à adapter la nouvelle de l'Autrichien et la sortie, deux fois différée, du film, tel qu'on peut le voir aujourd'hui. Plus de deux ans de tournage furent nécessaires entre novembre 1996 et la fin de l'année 1998. Le film sortit le 16 juillet 1999 aux États-Unis et le 15 sep-

31. Scénario de Frederic Raphael et Stanley Kubrick d'après Traumnovelle d'Arthur Schnitzler; image, Larry Smith; assistant, Leon Vitali; décors, Les Tomkins, Roy Walker; montage, Nigel Galt; musique, Jocelyn Pook; interprétation, Tom Cruise, Nicole Kidman, Sidney Pollack, Marie Richardson, Vinessa Shaw, Rade Sherbedgia, Todd Fiels; distribution, Warner Bros, durée 157 mn.

32. 1862-1931. 
tembre de la même année en France. Cette ultime production d'un cinéaste consacré comme un des grands maîtres du septième art des quarante dernières années a été couronnée par un large succès public même si les critiques ne furent pas unanimes ${ }^{33}$. De la mort du cinéaste, cinq jours après la sortie du film, événement qui contribua sans doute à son succès, on ne retint que les formules hagiographiques d'un journalisme de circonstance. Considérons malgré tout que dans la perspective qui est ici la nôtre, le sujet abordé par Eyes Wide Shut résonne comme le témoignage ultime d'un ressort essentiel du comportement humain, témoignage d'un artiste qui se sait au bord de la mort. Il raconte tout à la fois une, voire deux obsessions sexuelles (masculine et féminine), évoque la jalousie, la question de la norme et de la loi, des fantasmes et de la culpabilité qui les accompagne, des pulsions humaines, et d'une manière générale, de toutes les barrières mentales qui emprisonnent dans ce domaine les hommes comme les femmes.

Eyes wide shut met en scène un jeune couple new-yorkais, Bill et Alice Harford (Tom Cruise et Nicole Kidman, mari et femme au cinéma comme à la ville); lui médecin de la grande bourgeoisie, et elle, ex-directrice d'une galerie d'art. Après une soirée ou l'un et l'autre reçoivent les avances (appuyées) de deux jeunes mannequins pour Bill, et d'un séducteur hongrois (caricatural) pour Nicole, ils s'interrogent, rentrés à la maison, sur la question de leur désir intime. Bill croit Nicole incapable de le tromper tandis qu'elle fait voler en éclat cette belle certitude en lui avouant l'irrépressible désir qu'avait suscité en elle un marin, rencontré par hasard lors d'un voyage où leur amour ne faisait pourtant aucun doute ${ }^{34}$.

Cette révélation entraîne pour Bill une prise de conscience douloureuse. La suite du film montre le cheminement nocturne de Bill dans la ville. Son itinéraire l'emmène tout d'abord au chevet d'un moribond où il reçoit les avances de la fille du défunt. Il se fait ensuite bousculer et désigner comme homosexuel par des jeunes gens aux manières de ceux d'Orange mécanique. Il accepte ensuite de "monter» chez une prostituée, mais un appel de sa femme l'empêche de mener à bout cette expérience. Revenant le lendemain chez la jeune prostituée, il apprend qu'elle est atteinte du sida. Puis c'est une fillette qui le sollicite dans un lieu étrange où il vient louer un costume et un masque afin de pénétrer clandestinement, grâce à un ami, dans un château. Dans ce lieu qui figure son inconscient, se déroule, tous les convives étant masqués, une soirée orgiaque sous l'égide d'un grand ordonnateur. Mais Bill, identifié comme indésirable (la loi, la norme), est chassé de ce lieu de fantasmes masculins réservé à ceux, tenant de la morale et de l'ordre bourgeois, qui ont pac-

33. Le film avait rapporté au 6 novembre 2000, hors marché américain, 105 millions de dollars et occupait à cette date la 171è place au Top grossing movies of all time at the international box office.

34. Cf. infra, la retranscription de la scène. 
tisé avec le désir et la culpabilité. Ce lieu de rêve éveillé révèle son imposture (il n'a pas le droit d'y entrer), et le ramène à la réalité par la mort d'une jeune prostituée - celle-là même qu'il avait sauvée au cours de la soirée chez les Ziegler - et qui, pendant la cérémonie, s'est sacrifiée pour lui au cours d'une mise en scène dont il ne sait quelle est l'exacte part de jeu. Il la retrouve morte d'une overdose à la morgue.

Au cours de chacune de ces rencontres, Bill est incapable de concrétiser ce qu'il croit être la revendication libératoire de sa pulsion sexuelle. Et ce, quelle que soit la nature de celle-ci, hétérosexuelle, homosexuelle, pédophilique, travestie. C'est ce travestissement qui figure l'impossibilité d'un regard (Eyes Wide Shut) qui empêche de voir la réalité par sa soumission à la norme et aux tabous, et où les tenants des bonnes mœurs sont cachés derrière des masques pour mieux débrider leur passion pour une vaine sexualité. Ziegler, qui participait à l'orgie, tente de convaincre Bill que tout cela n'était qu'un jeu. Mais la mort de la jeune fille laisse celui-ci circonspect. Au retour de cette nuit agitée, Bill retrouve sa femme endormie, son masque posé à sa place près d'elle sur le lit. Cette vision déroutante de sa propre image le fait pleurer. Nicole et Bill se retrouvent et se parlent, mais en dépit des mots échangés ${ }^{35}$, sans guère comprendre ce qui leur est arrivé. L'épilogue montre le couple dans un grand magasin, faisant avec leur fille des courses pour Noël. Finalement résignés l'un et l'autre à rentrer dans la norme de la conjugalité, et donc, selon Kubrick, de l'aveuglement.

C'est moins l'histoire labyrinthique qui est importante que cette déambulation d'un homme dont la révélation faite par sa femme attise la jalousie en même temps qu'elle le laisse perplexe et impuissant. Car il s'agit d'une jalousie sans rival réel, une jalousie des fantasmes de l'autre, autant dire une découverte de l'autre impossible à posséder. "L'erreur de Bill, a écrit un critique, c'est de penser que la pulsion n'est qu'irrationnelle et instinctive. Au moment où il se croit libre, sa volonté de contrôler le monde n'a jamais été aussi grande ${ }^{36}$ ". C'est bien de pouvoir qu'il s'agit, de l'univers grand bourgeois et lisse du médecin de Manhattan, à celui, impossible à assumer, que lui révèle son désir. Derrière Bill, le médecin au pouvoir tranquille - un pouvoir de vie et de mort sur ses semblables surgit un personnage ahuri qui découvre, à travers ses propres pulsions, la grande mascarade d'une humanité qu'il croyait pourtant connaître.

Il s'agit aussi de la revendication du désir féminin, qui fait voler en éclat les certitudes masculines, sans doute ici brossées de façon caricaturales, mais qui par cet aveu deviennent prétexte à un large panoramique

35. "J'ai enfin ouvert les yeux" explique Nicole à Bill alors qu'il ne s'agit que d'une résignation à un retour à sa vie antérieure.

36. SAADA, Nicolas, "Scène de l'envie conjugale», Cahiers du Cinéma, septembre 1999, n 538, p. 34 . 
sur les pulsions refoulées et inavouées d'une société toute entière; en même temps que l'inconscient traverse ce regard (toujours les yeux fermés) en lui proposant justement de s'affranchir de ces tabous.

Ce couple à l'écran est en quête permanente d'un désir impossible qui prend le pas sur les sentiments, des sentiments qui ressemblent à l'habitude dès lors que le désir se porte ailleurs. Le film est le récit de cette impossibilité de vivre son désir et celui de l'autre en dépit de sa revendication. Les exigences du désir font soit exploser l'équilibre conjugal (on rapprochera cette affirmation des $41 \%$ de divorces constatés après dix ans de mariage en France, un peu plus aux États-Unis); soit imposent un retour à la norme en fermant à nouveau les yeux parce qu'il n'y a guère de prolongement viable à cette prise de conscience. Si ce n'est l'aveuglement consenti, à une fuite à l'infini, à la manière de 2001 l'odyssée de l'espace.

Désir et fantasmes masculins pourchassés dans la quête du passage à l'acte jamais réalisé ou assouvi s'oppose dans Eyes Wide Shut au désir foudroyant féminin, secret et accepté dans sa forme d'impossible. Le monde masculin associé à la quête sans fin de l'assouvissement sexuel contre le retranchement féminin dans l'imaginaire relève d'un cliché que Kubrick sait utiliser en le déformant comme pour mieux le dénoncer. Le choix même de Tom Cruise, qui n'exprime guère de sensualité, dépassé qu'il est par les événements, impuissant face aux sollicitations dont il est l'objet et qui refuse le passage à l'acte, montre à quel point Kubrick se joue de ce cliché en utilisant une star masculine du cinéma à l'inverse de ce qui justement fait son succès. Le jeu avec les genres se résume ici par l'oxymoron du titre du film ("des yeux grand fermés») qui, comme le résume Samuel Blumenfeld, "décrit le conflit entre un homme impliqué dans une histoire à laquelle il ne comprend rien alors que sa femme apparaît omnisciente, sachant tout sans rien avoir vu ${ }^{37}$ ".

Dans l'un et l'autre cas, les deux sexes se rejoignent dans cet espace freudien où l'assouvissement de la sexualité est appréhendée selon le désir au moins autant que selon les actes. La force de Kubrick est de montrer que c'est sans doute là le vrai danger qui guette le couple. Bien plus que l'adultère, la seule force du désir d'un(e) tiers interdit à l'autre un espace secret, ce que n'importe quelle liaison extra-conjugale consommée laisse malgré tout à sa portée. «En ce sens, explique Élisabeth Roudinesco, Kubrick frappe très fort (car) il réactualise le désir en tant que tel, beaucoup plus menaçant que dans sa réalisation ${ }^{38}$. Cette mise en scène de l'imaginaire est organisée dans

37. "Le mystère du couple ou l'enfer selon Kubrick", Le Monde du 15 septembre 1999.

38. Élisabeth Roudinesco, entretien avec Louis Guichard, "Kubrick rétablit la puissance du fantasme», Télérama, 15 septembre 1999, p. 16. La psychanalyste souligne également que le film met aussi en avant "cette idée de Freud selon laquelle certaines névroses, voire certaines grandes folies pouvaient trouver leur origine non dans un 
un vaste labyrinthe: parcours initiatique consécutif à la prise de conscience de Bill, dédale d'images rêvées, cloisonnement des dialogues échangés par le couple qui ne se comprend pas, montage parallèle qui sépare les deux époux après la scène de la dispute, effets de miroir aveuglant le regard que portent les personnages sur eux-mêmes, jeu chromatique mêlant et opposant le chaud et le froid ${ }^{39}$, et bien sûr, écho des références aux œuvres précédentes du réalisateur. De ce labyrinthe révélé par le désir émerge une réflexion philosophique entre l'intime et l'universel, une sorte d'ultime témoignage sur l'humanité d'un artiste au bord de la mort. Ainsi, comme l'explique Nicolas Saada, contrairement à 2001 l'odyssée de l'espace, Orange mécanique ou Barry Lyndon, "films dans lesquels un événement isolé débouchait sur une réflexion philosophique [où] l'on passait [...] de l'infiniment petit à l'infiniment grand $[\ldots]$ ici, la dernière grande aventure du siècle n'est autre que nous-même. Il serait dérisoire d'essayer de voir dans nos pulsions l'expression d'une liberté d'action. Bien au contraire, elles expriment notre attachement aux idées et au monde, notre soif de contrôle ${ }^{40}$ ".

Mais au-delà de cette quête d'un pouvoir absolu, le film résonne étrangement à un siècle d'intervalle avec cette société viennoise $a$ priori si différente de la société occidentale d'aujourd'hui. En effet, quoique la libéralisation des mœurs ait à l'évidence transformé, en la banalisant, l'éternelle question de l'adultère dans la Babylone new-yorkaise, cela ne change rien. La culpabilité et son escorte de fantasmes n'a pas pour autant disparu. Le verdict de la psychanalyse est formel: "la révolution sexuelle a libéré les mœurs, mais non les consciences ${ }^{41}$ ». Et face à cette modernité amoureuse, issue de la techno-idéologie de la contraception, de la conscience et de la revendication du désir, de l'individualisme et du libéralisme conquérant, le film de Kubrick rappelle que le cocon des certitudes et de l'ennui peut se briser à tout moment. C'est peut-être cette idée d'implosion qui résonne, menace et fascine à la fois. Un jeu entre le pouvoir (sur l'autre, de l'autre) et le désir individuel qui, au prétexte d'un questionnement sur les aléas de la conjugalité, "nous confronte à cette catastrophe intérieure que nous redoutons tous, mais que nous attendons comme une délivrance ${ }^{42}$ ".

quelconque traumatisme réel du Sujet, mais dans sa seule construction imaginaire altérant son rapport au monde». (Ibidem.) Cette remarque à elle seule pourrait nourrir bien des réflexions sur la question de la perception et des représentations pour les historiens.

39. «Le héros kubrickien est soumis aux limites de son regard. Ne se contemplant au départ que dans un miroir (le regard se perdant dans le fait de se regarder lui-même), le héros n'arrive plus à voir au-delà de ce regard. La fuite vers l'infini présentée dans 2001, a space odyssey (1968) est alors transposée en un éternel retour sur soi, fermant le film en boucle et en confirmant son schéma circulaire et labyrinthique» écrit Bruno Cornellier, "Les limites du regard» Cadrage. Le magazine du cinéma international, édité en ligne www.Cadrage.net.

40. Ibidem.

41. Élisabeth Roudinesco, op. cit.

42. SAADA, Nicolas, "Scène de l'envie conjugale», Cahiers du Cinéma, septembre 1999, $\mathrm{n}^{\circ} 538$, p. 34 . 


\section{Extrait}

Eyes Wide Shut.

Troisième scène. Intérieur/nuit.

Le lendemain de la soirée chez Ziegler, Bill et Alice Harford, chez eux, dans leur chambre éclairée par des couleurs chaudes tandis que de la fenêtre émane une lumière froide bleue. Alice roule un joint de marijuana qu'elle fume avec Bill. Tous deux sont déshabillés, sur le lit. Bob demeure assis sur le lit durant toute la scène, tandis qu'Alice, tout d'abord près de lui, se déplace ensuite dans la chambre.

Alice: «Dis-moi, j'aimerais savoir, ...les deux filles à la fête hier soir, ... est-ce que par hasard ça n'a pas fini par une baise?

Bob: Quoi? Mais enfin, de quoi tu parles?

Alice: Oh..; je te parle tout simplement des deux filles à qui tu faisais si brutalement du rentre-dedans.

Bob: Je n'ai fait aucun rentre-dedans à personne.

Alice: Qui c'étaient?

Bob: deux filles comme on en voit mille ... des mannequins.

Alice: Et où as-tu disparu avec elles pendant des heures?

Bob: $m m$... attends un peu, attends... (l'embrassant) j'ai jamais disparu, (plan rapproché sur Bob et Alice assis sur le lit) avec qui que ce soit. Ziegler ne se sentait pas bien, on m'a dit de monter l'ausculter. Et toi c'était qui ce type avec qui tu dansais?

Alice: (riant) un vieil ami des Ziegler.

Bob: Qu'est-ce qu'il voulait?

Alice: Qu'est-ce qu'il voulait? Oh qu'est-ce qu'il voulait .... mmm... baiser, première étape: baiser et de toute urgence.

Bob: (amusé) et c'est tout?

Alice: oui, oui c'était tout.

Bob:il voulait juste baiser ma femme.

Alice: (riant)

Bob:je trouve ça compréhensible.

Alice: (se renfrognant en fumant le joint) compréhensible?

Bob: parce que tu es une très très très belle femme.

Alice: (se levant du lit) oh la attends, alors parce que je suis une très belle femme la seule raison qui pousse un homme à s'intéresser à moi, ... c'est qu'il a envie de me baiser... $\mathrm{mmm}$, c'est ça que tu me racontes? (plan rapproché de Bob)

Bob: (perplexe) Bon ce n'est, ce n'est pas aussi simpliste que çà, mais... (plan américain d'Alice qui s'est levée) je crois... on sait tous les deux à quoi nous en tenir sur les hommes.

Alice: Alors en partant de là, je dois en conclure (plan moyen de Bob assis sur le lit) que tu voulais te faire ces deux mannequins?

Bob: $Y$ a des..., y a des exceptions.

Alice: (plan américain d'Alice debout dans l'embrasure de la porte) Qu'est-ce qui fait de toi une exception?

Bob: (plan moyen de Bob assis sur le lit) ce qui fait de moi une exception c'est que... tout d'abord je suis amoureux de toi, ...que toi et moi nous sommes mariés, et puis surtout que... jamais je ne te mentirais, ou je ne te ferais souffrir.

Alice: (soufflant, plan américain, elle se déplace dans la pièce) Est-ce que tu te rends compte? Tout ça revient à dire que la seule raison pour laquelle tu ne t'es pas envoyé ces top models, c'est par simple considéra- 
tion pour moi? (plan moyen de Bob assis sur le lit). Et pas parce que tu n'avais pas envie d'elles?

Bob: Non là, on se calme Alice, ce pétard te rend trop agressive.

Alice: (plan américain d'Alice debout devant la fenêtre) Non ! C'est pas le pétard, c'est toi. (Bob assis sur le lit en plan rapproché) Pourquoi tu ne peux pas me répondre franchement merde?

Bob: Mais c'est précisément ce que j'avais l'impression de faire. Je sais même pas pourquoi on se dispute.

Alice: (plan américain d'Alice devant la fenêtre) On ne se dispute pas. Ce que je veux, c'est essayer de voir où tu te situes exactement. (Bob plan rapproché)

Bob: Où je me situe exactement? Bon.

Alice: (plan moyen d'Alice assise, qui se lève, elle se déplace dans la pièce, la caméra la suit en plan américain) Écoute, disons, disons par exemple que tu vas examiner une très belle femme. Elle est là, dans ton cabinet, à poil, et toi il faut que tu lui palpes les nichons. Ce que j'aimerais savoir, ...ce que j'aimerais savoir, c'est ce que tu as dans la tête au moment où tu tripatouilles la dame.

Bob: (plan moyen de Bob assis sur le lit) Alice, tu sembles l'oublier, je suis médecin. (geste fataliste d'Alice en plan américain). Tout ça c'est complètement impersonnel (plan moyen de Bob assis sur le lit) et en plus mon assistante est toujours présente.

Alice: (plan moyen d'Alice assise) Quand tu palpes des seins c'est rien de plus que du professionnalisme, c'est ça que tu dis en clair

Bob: (plan moyen de Bob assis sur le lit) Précisément. Le sexe est la dernière des choses qui me vient à l'esprit quand j'examine une patiente.

Alice: (plan moyen d'Alice assise sur le tabouret) Et elle, pendant qu'elle se fait tripoter les ninis, tu crois pas qu'elle a l'occasion de fantasmer sur le si beau docteur Hartford.

Bob: (plan moyen de Bob assis sur le lit) Je t'en prie, je t'assure que le sexe est la dernière chose à laquelle penserait cette putain de bonne femme inventée de toute pièce.

Alice: (plan moyen d'Alice assise) Comment peux-tu en être si sûr?

Bob: (plan moyen de Bob assis sur le lit) Déjà pour une bonne raison, elle panique rien qu'à l'idée de ce que je peux découvrir.

Alice: (plan moyen) D'accord, d'accord, mais une fois que tu lui as dit que tout va bien, alors quoi?

Bob: (plan moyen) Alors quoi? euh, je sais pas Alice euh...(tapant dans ses mains), alors quoi? euh ..., écoute..., les femmes? ... elles ne ..., bref, en général, elles ont une autre vision des choses.

Alice: (fixant Bob en se relevant et le montrant du doigt, la caméra la suit en plan américain) Des millions d'années d'évolution pour çà ! Pour çà ! Les mecs, ils faut absolument qu'ils baisent tout ce qui bouge mais pour les femmes, pour elles on ne pense plus que sécurité, engagement (plan rapproché de Bob), toutes ces conneries quoi !

Bob: C'est caricatural comme point de vue ce que tu dis mais oui il y a un peu de çà.

Alice: (exaspéré, plan américain) Si vous les hommes vous saviez ce que nous ...

Bob: (plan rapproché) Je vais te dire ce que je sais: tu es raide défoncée ce soir, t'as envie qu'on se dispute et par dessus le marché tu essayes de me rendre fou de jalousie.

Alice: (plan américain) Mais toi la jalousie ce n'est pas ton genre. 
Bob: (plan rapproché) Non effectivement.

Alice: (plan américain)Tu n'as jamais été jaloux à cause de moi n'est-ce pas?

Bob: (plan rapproché) Non c'est vrai.

Alice: (s'énervant, plan américain) Et pourquoi tu n'as jamais été jaloux à cause de moi?

Bob: (plan rapproché, légère plongée) Mais je n'en sais rien Alice ! Peut-être parce que tu es ma femme ! peut-être parce que tu es la mère de mon enfant et que je sais que tu es incapable de me tromper.

Alice: (plan américain, elle se déplace devant la fenêtre) Tu es vraiment, vraiment sûr de toi n'est-ce pas?

Bob: (plan rapproché, légère plongée) Non, je suis sûr de toi.

Alice: (se tordant de rire, la caméra la suit)

Bob: (plan rapproché) Tu trouves çà si marrant

Alice: (riant de plus belle elle tombe par terre, la caméra la suit)

Bob: (plan rapproché de Bob) Putain ! Putain de fou rire merde !

Alice: (continuant à rire puis se calmant, plan rapproché) tu te souviens.. tu te souviens de ces vacances cet été qu'on a passé à Cape Code? Bob: (plan moyen) Oui.

Alice: (plan moyen elle est assise par terre) Alors tu te souviens peutêtre d'un soir où nous étions dans la salle à manger et il y avait un jeune officier de marine, et il était à côté de notre table avec deux autres officiers.

Bob: Non.

Alice: On est venu lui apporter un message. Il est parti juste après. (la musique monte crescendo) Tout çà n'évoque rien pour toi?

Bob: (plan rapproché) Non.

Alice: (plan moyen) Eh bien ... je l'avais déjà vu une fois le matin à la réception. Il venait de remplir sa fiche au comptoir de l'hôtel, il suivait le chasseur qui portait sa valise, jusqu'à l'ascenseur. D'un regard il m'a détaillée en passant, un simple regard... rien de plus, ... mais ...je pouvais à peine bouger (gros plan sur Bob inquiet, puis sur Alice en plan moyen) Cet après-midi là, Helena est allée au cinéma avec une copine, et toi et moi, nous avons fait l'amour... Nous (gros plan large de profil d'Alice) avons fait plein de projets pour l'avenir et nous avons parlé d'Helena. N'empêche que pendant tout ce temps, c'est lui, et lui seul (gros plan de Bob) qui a hanté mes pensées (gros plan large Alice de face). Et j'ai pensé que si jamais il voulait de moi, même si ça n'était qué pour une nuit, j'étais prête à laisser tomber (gros plan de Bob) ce qui était ma vie: toi, Helena, et tout mon avenir avec vous. Toute ma vie.(gros plan de _face d'Alice). Et ce qui était le plus étrange c'est qu'en même temps, tu comptais pour moi plus que jamais. Et à cet instant précis mon amour pour toi était à la fois tendre, triste (gros plan de Bob, puis Alice). J'ai à peine dormi cette nuit là. Quand je me suis réveillée au matin je paniquais. Je ne savais plus si j'avais peur qu'il ait quitté la ville ou si j'avais peur qu'il soit en bas dans le hall. Après, au dîner je me suis rendu compte qu'il était parti. Et ça a été un véritable soulagement (plan rapproché sur Bob, le regard fixe)."

Le téléphone sonne, Bob est appelé au chevet d'un patient défunt. (Fin de la scène) 


\section{Enchaînements}

Ces deux exemples montrent le chemin parcouru au cinéma dans l'expression des rapports de genre et de pouvoir. Que les temps aient changé, et que la forme ait évolué, nul n'en disconviendra. Il n'est néanmoins pas certain que la revendication du désir, enserrée par la censure, n'ait pas trouvé dans certains moments de grâce des formes de contournement plus habiles voire plus amusantes. L'œuvre de Hitchcock regorge d'allusions, habiletés et autres clins d'œil conçus dans ce dessein ${ }^{43}$. Les deux exemples choisis ici montrent tout d'abord que c'est au cœur de leur qualité d'œuvre qu'ils traitent le sujet. L'un juste après la Seconde Guerre mondiale, l'autre aujourd'hui. Mais ce qui les séparent n'est sans doute pas le plus remarquable. Dans ces deux films, la question du pouvoir, qu'il s'agisse des sentiments ou du désir représente un élément central qui échappe à des contextes séparés de plus de cinquante ans. À l'enchaînement explicite de Notorious sur fond de guerre larvée, et d'une certaine manière, justifié par le contexte et son genre de film d'espionnage, répond un faux déchaînement ( $a$ priori celui du désir qui ose s'exprimer) mais qui est tout sauf une évolution et encore moins une libération des rapports de genre. Eyes Wide Shut montre à quel point la libération des pulsions, n'est qu'une autre manière de garder les yeux fermés.

Il serait évidemment vain de vouloir repérer les films qui ont marqué de leur sceau la question du pouvoir et des genres dans leurs interactions sentimentales ${ }^{44}$. Mais l'omniprésence de cette thématique au cœur même des images du grand écran s'inscrit en filigrane dans un univers imaginaire où le cinéma rappelle souvent à l'histoire l'existence d'un discours parallèle: un souffle de fausses vérités dispensées par des images à la fois au cœur d'une époque et en dehors du temps. Il va de soi que ce qui a marqué la rupture la plus visible entre ces deux films est sans doute la déliquescence si ce n'est l'abolition de la censure ${ }^{45}$. Dans ce domaine, les

43. Citons l'exemple fort connu du plan final du train entrant dans le tunnel de North by Northwest (La mort aux trousses, Alfred Hitchcock, 1948), qui suit le plan du baiser échangé entre Cary Grant et Eva-Marie Saints dans le wagont-lit qui les emmène en voyage de noce.

44. Rappelons quelques titres qui, à l'origine, devaient être inclus ici. Rashomon (Akira Kurosawa, 1951) qui croise trois regards (2 masculins et un féminin) à propos d'un viol; Viridiana, 1961; El, 1952 et Belle de jour (1967, Luis Bunuel), qui expriment, avec le génie de Bunuel, l'impossible quadrature de la différence des sexes et de leurs rapports névrotiques: perversion, jalousie, domination, hypocrisie bourgeoise, etc. Soulignons également le cas d'une œuvre unique comme La maman et la putain (Jean Eustache, 1973), véritable pierre de touche du cinéma des années post-soixante-huit, qui en dit sans doute plus sur son époque que la plupart de ce qui a été écrit; ce qui fit dire de Jean Eustache qu'il avait su «bourrer la pellicule de l'air du temps, de ce qui domine, de ce qui est refoulé" (ACCURSI, Daniel, "cinéma érotique», in A. et O. VIRMEAU, Dictionnaire du cinéma mondial, op. cit., p.309).

45. On sait néanmoins que certaines images furent modifiées dans la version américaine de Eyes wide shut de sorte que soient gommées les parties génitales trop visibles. Le film fut classé R 17 aux États-Unis, c'est-à-dire autorisé au jeunes gens de moins de 17 ans accompagnés d'un adulte. 
films érotiques ou pornographiques marquent, dans les années soixantedix, un tournant dans la représentation du désir, de la suggestion à la reconstitution réelle ${ }^{46}$. Mais ne s'agit-il pas seulement, comme le propose Daniel Accursi, d'un simple "glissement progressif et inouï de la métaphore au réel ${ }^{47}$ ?». Le chef-d'œuvre dans ce registre, L'empire des sens (Nagisha Oshima, 1976) sut démontrer que le problème résidait moins dans la censure que dans l'usage et dans le sens qui pouvait s'exprimer grâce à cette liberté.

\section{Genre et pouvoir en cinéma}

Les genres bousculés dans la passion qui les anime au cinéma ne peuvent guère supporter une analyse qui s'appuierait sur les seuls rapports sociaux de sexe. La dénonciation des inégalités et plus généralement l'efficacité de l'analyse de la domination d'un genre par l'autre qui fonde une bonne partie des études féministes paraît ici insuffisante, ou réductrice.

En matière d'art, la morale égalitariste comme l'inversion du rapport de pouvoir entre homme et femme amène à une impasse. L'artiste Nancy Grossman par exemple en fit au milieu du siècle une forme de démonstration. Elle joua sur l'inversion en utilisant des images érotiques voire pornographiques mettant en scène ordinairement des femmes et en les remplaçant par des hommes (cf. buste d'homme ligoté vêtu de cuir, 1940). Mais l'inversion ne règle rien: "Elle rappelle nos différences sexuelles, écrit Anne Higonnet, mais ne les abolit pas. Il [le buste] est à la fois repoussant et fascinant, ce qui nous oblige à admettre que notre perception de la sexualité est liée aux signes du pouvoir, qu'on l'exerce ou qu'on y soit soumis ${ }^{48}$."

Devons-nous en conclure que la force des sentiments demeure, dans l'alchimie secrète du désir, impossible à arpenter pour l'historien? Sauf peut-être, en se risquant en dehors de son territoire balisé, c'est-à-dire en prenant le risque d'ausculter l'œuvre et ses secrets. Car le cinéma parce qu'il projette à l'infini cette question du pouvoir et du désir, en restitue avec force l'insondable vérité. Cette forme de vérité du cinéma, associée à la question centrale de l'amour a quelque chose de spécifique dans sa manifestation. Dans l'expérience singulière de l'œuvre comme dans celle de l'amour naissant, il n'y a guère d'apprentissage possible. La magie de l'œuvre comme celle de l'expérience amoureuse renvoient à un domaine inconnu. Sans doute pourrait-on appliquer ici

46. 23 avril 1975, date du premier film hardcore autorisé en France (Anthologie du plaisir, Alex de Keuzy, US).

47. ACCURSI, Daniel, "Érotique (cinéma)", in A. et O. VIRMEAuX (sld), Dictionnaire du cinéma mondial, éd. du Rocher, p. 305.

48. Higonnet, Anne, "Femmes, images et représentations" in DuBY, Georges et PERROT, Michelle, Histoire des femmes tome V, op. cit., p. 362. 
l'aphorisme de Gilbert Durand selon lequel "L'image pas plus que la vie ne s'apprend: elle se manifeste ${ }^{49}$ ".

Feu du désir, des sentiments et de la sexualité conjugués en une énergie imprévisible et toujours inquiétante pour l'ordre social. C'est la force de cette vérité que "les interdits tentent de contenir, pour que le monde réglé par le travail, la vie attelée, soit à l'abri du désordre qu'introduisent la mort et la sexualité ${ }^{50}$ ". C'est donc dans l'égarement et la démesure que se révèlent les rapports de pouvoir qui nous intéressent ici, un lieu de vertige où s'abîment les genres. Sans morale et sans justice. Sans que les théories humaines, fussent-elles féministes, puissent y avoir quelque influence ou proposer quelque solution. Le cinéma alimente et restitue l'imaginaire du seul lieu d'aventure et de conflit toujours intact aujourd'hui, qui oppose et rassemble hommes et femmes depuis leur origine. Il ne cesse de rejouer en images cette fantasmatique de l'autre dans le sentiment amoureux jusqu'à sa forme paroxystique où à force de désir, c'est la mort qui se profile. Il donne valeur d'explication à la revendication de Georges Bataille lorsqu'il écrivait: "Ce que nous voulons est ce qui épuise nos forces et nos ressources et qui met, s'il le faut, notre vie en danger ${ }^{51}$." Sujet inépuisable de plaisirs et de tourments, d'engagement total du Sujet, puissante énergie secrète de la dialectique intime entre les genres, l'amour traduit en ces termes demeure un mystère à la seule portée de la beauté de l'art. Car si l'esthétique témoigne de sa manifestation, elle nous laisse démunis quant à son élucidation.

La recherche séculaire de l'harmonie entre homme et femme n'est-elle pas tributaire de ce même questionnement?

49. Les structures anthropologiques de l'imaginaire, Dunod, $19691^{\text {re }}$ éd., 1992, p. 477.

50. Francis MARMANDE, «George Bataille», Encyclopédie Universalis, t. 3, p. 897.

51. Cité par VIRMAUX, op. cit., p. 309. 


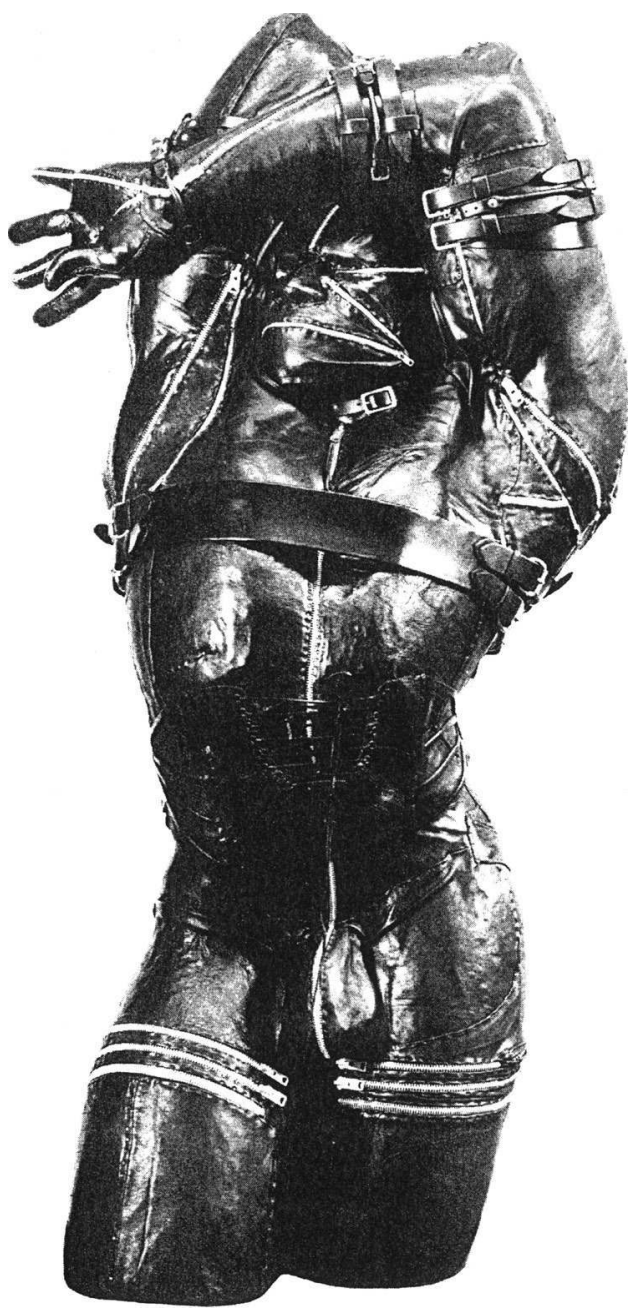

Nancy Grossman, "Buste d'homme», Sculpture de bois habillée de cuir avec fermeture Éclair, 1.91 m, 1971., Jérusalem, Israel Museum. 


\section{RÉSUMÉ}

L'objet de cet article est de montrer pour le second vingtième siècle la grâce de l'art cinématographique dans la traduction des sentiments, du désir et des rapports de pouvoir qui en sont issus. De l'après-guerre des Enchaînés d'Alfred Hitchcock (1946) à la fin de siècle d'Eyes Wide Shut de Stanley Kubrick (1999), il s'agit moins de baliser un demi siècle de pouvoir sur le thème de l'amour et du pouvoir au cinéma que d'évoquer, à travers ces deux exemples, l'écriture filmique des sentiments et du désir comme lieu de pouvoir sexué. Tout en restituant à l'œuvre de cinéma sa force singulière.

\section{ABSTRACT}

The purpose of this article is to demonstrate for the second half of the twentieth century, the grace of cinematographic art in its treatment of sentiments, of desire and of the resulting balances of power. From Alfred Hitchcock's post-war Notorious (1946) to Stanley Kubrick's end-of-century Eyes Wide Shut (1999), the issue is not so much to view half a century of power concerning the theme of love and of power of the cinema, rather it is to evoke, using these two examples, the cinematic writing of sentiments and desire as a scene of sexual power. All the while restoring to the art of cinema its unique force. 